\title{
Análisis morfotectónico y gravimétrico en un valle intermontano de la Precordillera Central de San Juan, Argentina
}

\author{
L.P. Perucca ${ }^{1}$ \\ F. Audemard M. ${ }^{2}$ \\ J.M. Alcacer Sánchez ${ }^{3}$ \\ M. Rothis ${ }^{4}$ \\ M. Vargas $^{5}$ \\ F. Haro $^{6}$ \\ F. Tejada \\ P. Blanc ${ }^{8}$ \\ N. Vargas ${ }^{9}$ \\ G. Lara $^{10}$ \\ M. Onorato ${ }^{11}$
}

Recibido el 8 de marzo de 2019; aceptado el 5 de julio de 2019

\section{Resumen}

En este trabajo se efectúa un análisis morfotectónico y gravimétrico de la cuenca del río Talacasto, en Precordillera Central, con énfasis en el río de La Burra. Se describen además las principales estructuras con evidencia de actividad

Gabinete de Neotectónica y Geomorfología (INGE0), Facultad de Ciencias Exactas, Físicas y Naturales, Universidad Nacional de San Juan (FCEFyN-unss), Argentina. Centro de Investigaciones de la Geósfera y Biósfera (CIGEOBIO-CONICET), Argentina. Correo electrónico: Iperucca@unsj-cuim.edu.ar

2 Funvisis Earth Sciences Dept., Venezuelan Foundation for Seismological Research, Venezuela. Correo electrónico: faudemard@gmail.com

3 Gabinete de Neotectónica y Geomorfología (INGGo), Facultad de Ciencias Exactas, Físicas y Naturales, Universidad Nacional de San Juan (FCEFyN-uns)), Argentina. Centro de Investigaciones de la Geósfera y Biósfera (CIGEOBIO-CONICET), Argentina. Correo electrónico: alcacersanchez@gmail.com

4 Gabinete de Neotectónica y Geomorfología (INGEo), Facultad de Ciencias Exactas, Físicas y Naturales, Universidad Nacional de San Juan (FCEFyN-unss), Argentina. Centro de Investigaciones de la Geósfera y Biósfera (CIGEOBIO-CONICET), Argentina. Correo electrónico: marothis@gmail.com

5 Gabinete de Neotectónica y Geomorfología (INGG0), Facultad de Ciencias Exactas, Físicas y Naturales, Universidad Nacional de San Juan (FCEFyN-unss), Argentina.

6 Gabinete de Neotectónica y Geomorfología (INGGo), Facultad de Ciencias Exactas, Fisicas y Naturales, Universidad Nacional de San Juan (FCEFyN-unss), Argentina. Centro de Investigaciones de la Geósfera y Biósfera (CIGEOBI0-CONICET), Argentina. Correo electrónico: federicoharo58@gmail.com

7 Gabinete de Neotectónica y Geomorfología (INGE0), Facultad de Ciencias Exactas, Físicas y Naturales, Universidad Nacional de San Juan (FCEFyN-uns), Argentina. Centro de Investigaciones de la Geósfera y Biósfera (CIGEOBIO-CONICET), Argentina. Correo electrónico: flaviatejada@gmail.com

8 Gabinete de Neotectónica y Geomorfología (INGGo), Facultad de Ciencias Exactas, Fisicas y Naturales, Universidad Nacional de San Juan (FCEFyN-unss), Argentina. Centro de Investigaciones de la Geósfera y Biósfera (CIGEOBIO-CONICET), Argentina.Correo electrónico: pabloandresblanc@gmail.com

9 Gabinete de Neotectónica y Geomorfología (INGGo), Facultad de Ciencias Exactas, Físicas y Naturales, Universidad Nacional de San Juan (FCEFyN-uns), Argentina.Correo electrónico: nicolasvargas2003@yahoo.com.ar

10 Gabinete de Neotectónica y Geomorfología (INGE0), Facultad de Ciencias Exactas, Físicas y Naturales, Universidad Nacional de San Juan (FCEFyN-unss), Argentina. Correo electrónico: gabyge021@hotmail.com

11 Gabinete de Neotectónica y Geomorfología (INGE0), Facultad de Ciencias Exactas, Físicas y Naturales, Universidad Nacional de San Juan (FCEFyN-unss), Argentina. Correo electrónico: onoratomariaromina@gmail.com 
tectónica cuaternaria. La morfología irregular de la cuenca y la diversidad de depósitos aluviales encontrados a lo largo del río de La Burra (colector principal de la cuenca) permitieron inferir que la misma se encuentra en un ambiente tectónico activo con fuertes controles litológicos y estructurales. Las principales fallas descritas se ubican en el piedemonte oriental de la Sierra de La Crucecita, y occidental de la sierra de Talacasto ( $30^{\circ} 54^{\prime}-31^{\circ} \mathrm{S}$ y $68^{\circ} 47^{\prime}-68^{\circ} 55^{\prime} \mathrm{O}$ ), unos $70 \mathrm{~km}$ al noroeste de San Juan, en el Departamento Ullum. Las mismas se denominaron de norte a sur: Las Crucecitas y Vertientes (en el piedemonte oriental de la sierra de La Crucecita) y el sistema de falla Talacasto occidental (piedemonte occidental de la sierra homónima). Las fallas afectan depósitos asignados al Pleistoceno tardío y Holoceno y muestran en las exposiciones naturales identificadas, rasgos típicos de ambientes compresivos y que evidencian su actividad cuaternaria. Las estructuras identificadas coinciden con el estilo estructural de tectónica de piel delgada de vergencia oriental, característico de Precordillera Central. Además, se identificó un control estructural sobre el río de La Burra, con la generación de al menos tres terrazas de erosión sobre su margen izquierda. Esta deformación se vincula con el tectonismo activo que a escala regional afectó y afecta al ámbito precordillerano. Finalmente, se identificaron las principales estructuras geológicas a partir del análisis e interpretación de las anomalías gravimétricas, empleando datos obtenidos de WGM12 (World Gravity Map, 2012), que incluye datos de gravedad terrestre y satelital derivados del modelo EGM2008. La respuesta gravimétrica del basamento que compone el área en estudio, muestra una correlación con la estructura superficial y podría estar asociada a los diferentes dominios morfotectónicos reconocidos en la región.

Palabras clave: cabalgamiento, vergencia oriental, piel delgada, geoformas, La Burra, Talacasto, Cuaternario.

\section{Abstract}

In this work, we have made a morphometric analysis of the Talacasto river basin (Central Precordillera), with emphasis on the La Burra River. The main structures with evidence of Quaternary tectonic activity are also described. The irregular morphology of the basin and the diversity of alluvial deposits found along the La Burra River allow the authors to infer that it is in an active tectonic environment with strong lithologic and structural controls. These main structures are located in the eastern piedmont of the Sierra de la Crucecita, and western piedmont of the sierra de Talacasto ( $30^{\circ} 54^{\prime}-31^{\circ} \mathrm{S}$ and $68^{\circ} 47^{\prime}-68^{\circ} 55^{\prime} \mathrm{W}$ ), about $70 \mathrm{~km}$ northwest of San Juan, in the Ullum Department. The faults located in both piedmonts are named from north to south: Las Crucecitas and Vertientes (in the eastern piedmont of the La Crucecita range) and the Western Talacasto fault system (in the western piedmont of the homonymous range). The faults affect alluvial deposits assigned to the Late Pleistocene and Holocene and show typical features of ongoing compressional Quaternary tectonic activity in the evaluated 
natural exposures. Faults exhibit the east-verging thin-skinned structural style typical of Central Precordillera. This deformation is related to the regional tectonics that affected and currently affects the Precordilleran region. Finally, the main geological structures were identified by the analysis and interpretation of gravimetric anomalies, employing data obtained from WGM12 (World Gravity Map 2012), which includes earth and satellite gravity data derived from the EGM2008 model. The gravimetric response of the crustal blocks that compose the study area shows a correlation with the outcropping crustal structure and could be associated to the different morphotectonic domains recognized in the region.

Key words: thrusting, eastern vergence, thin-skinned tectonics, landforms, La Burra, Talacasto, Quaternary.

\section{Introducción}

El área de estudio se ubica en el centro de la Provincia de San Juan, Argentina, entre los $30^{\circ} 54^{\prime}$ y $31^{\circ} 00^{\prime} S$ y $68^{\circ} 55^{\prime}$ y $68^{\circ} 45^{\prime} \mathrm{O}$, Departamento de Ullum, a unos $70 \mathrm{~km}$ al noroeste de la capital de San Juan (Figuras 1a, b). La zona corresponde a la provincia geológica de Precordillera Central, en el borde oriental de la sierra de La Crucecita y occidental de la sierra de Talacasto. Los ríos y arroyos ubicados en los piedemontes de ambos cordones montañosos drenan hacia el sur, a partir de una divisoria de aguas ubicada al oeste del bolsón de Gualilán, por el río de La Burra hasta el río Talacasto (Figura 1c). Las deformaciones cuaternarias identificadas en ambos piedemontes modifican la red de drenaje, ya que los pequeños arroyos tributarios son susceptibles a las variaciones, aún las sutiles de la topografía, en función de su tasa de erosión (Audemard, 1999).

El diseño del drenaje en el área de estudio es el resultado de una combinación de factores climáticos, tectónicos, litológicos, geomorfológicos, de suelo y vegetación. Este patrón no sólo responde a las condiciones presentes, sino que hereda las condiciones de modelados anteriores. Las diferencias identificadas en el drenaje se deben a la combinación de estos factores y al predominio de uno de ellos. Por ello, las características de la red de drenaje en el río Talacasto varían de acuerdo con el sector analizado, respondiendo principalmente a controles geológicos y geomorfológicos. Los ríos son de carácter efímero y solamente llevan agua durante las lluvias torrenciales que ocurren esporádicamente durante los meses de verano del hemisferio austral (diciembre-marzo).

Existen en el área muy escasos antecedentes referidos a estructuras neotectónicas en la depresión. En tal sentido, Casa et al. $(2011$, 2014) mencionaron algunas estructuras en el piedemonte occidental de la sierra de Talacasto, aunque no indicaron su cinemática ni los niveles aluviales afectados. De igual manera, Perucca y Vargas (2014) describieron una exposición natural ubicada 

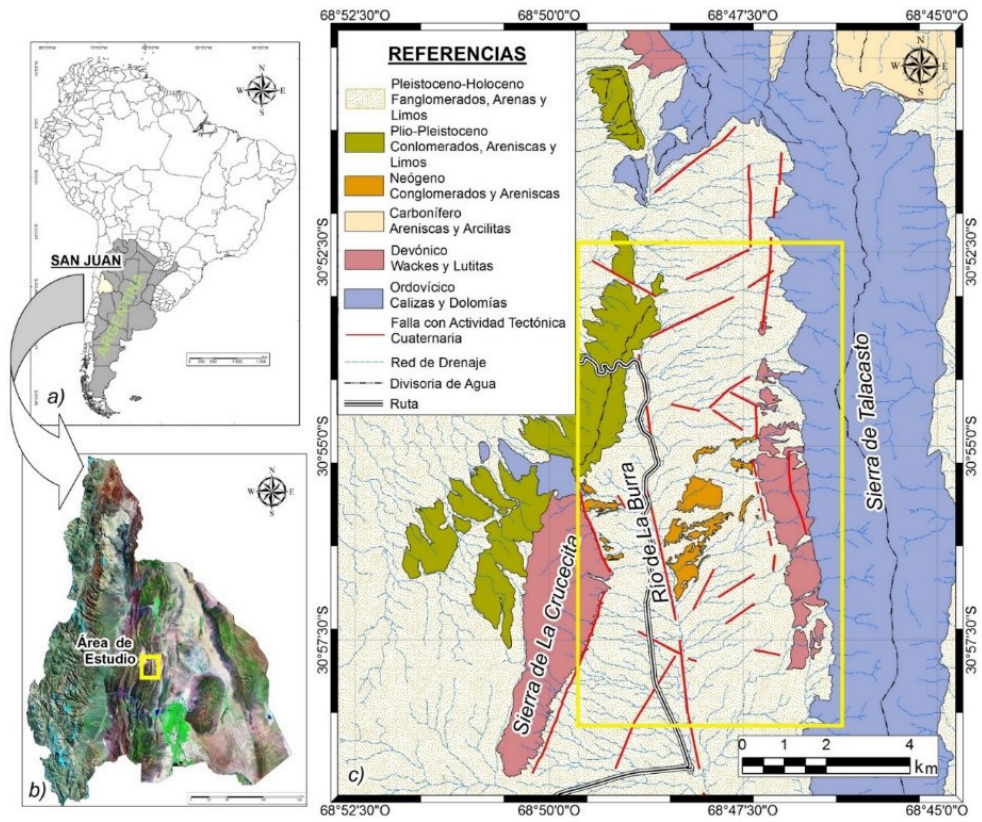

Figura 1. a y b) Ubicación del área de estudio en San Juan y Argentina, c) Geología del área de estudio (modificado de Furque et al., 1998). El recuadro identifica la ubicación de la figura.

en la porción norte del piedemonte oriental de la sierra de La Crucecita, en donde identificaron una falla inversa buzante al oeste a la cual denominaron falla Las Crucecitas, mientras Levina et al. (2014) evaluaron el relleno cenozoico del sector frontal de la cuenca de antepaís de Precordillera en tres quebradas ubicadas a lo largo del río San Juan (una de ellas, el río de La Burra) concluyendo que las tres sucesiones neógenas ubicadas en estas depresiones registran la construcción interna de Cordillera Frontal, el volcanismo de arco regional y la exhumación inicial de la Precordillera. Los cambios de procedencia registrados en la edad del zircón detrítico sugirieron un acortamiento inicial en la Cordillera Frontal que coincidió con el cambio de acumulación eólica a fluvial en la cuenca de antepaís adyacente, durante el Mioceno temprano (24-17 Ma) y consideraron entre los 12 y $9 \mathrm{Ma}$, como la probable edad de exhumación inducida por el empuje y levantamiento de la Precordillera (Levina et al., 2014).

Allis (2016), por su parte, analizó el piedemonte oriental de la sierra de la Crucecita e identificó estructuras con evidencia de actividad neotectónica, mientras Ocaña et al. (2017) presentaron un mapa geomorfológico del abanico aluvial del río Talacasto e indicaron algunos procesos geomorfológicos dinámicos y potencialmente destructivos para la infraestructura pública. 
En este trabajo se realizó un análisis morfométrico y morfotectónico de la cuenca del río Talacasto, con énfasis en el tributario del río de La Burra. Además se efectuó un análisis neotectónico en los piedemontes ubicados en el valle del río de La Burra, en los que se identificaron las principales fallas con actividad tectónica cuaternaria. Por otro lado, se intentó definir las estructuras geológicas del área basadas en el procesamiento y análisis de datos gravimétricos satelitales, los cuales tienen la ventaja de que permiten ver las variaciones de densidad en el subsuelo con buena estabilidad espacial y sin perturbaciones por aproximaciones matemáticas. De esta forma se intenta determinar si existe una correspondencia entre las unidades morfotectónicas identificadas en superficie y su continuidad en subsuelo basada en el procesamiento y análisis de los datos gravimétricos.

Se intenta de esta forma mejorar el conocimiento sobre las estructuras activas situadas en la depresión intermontana cercanas a la ciudad de San Juan (población > 700000 habitantes) y cerca de las principales presas hidroeléctricas de la región.

\section{Marco tectónico}

En el marco geotectónico actual de convergencia entre las placas de Nazca y Sudamericana, correspondiente al sector sur de Los Andes Centrales, el segmento de subducción comprendido entre los $28^{\circ}$ y los $33^{\circ}$ de latitud sur, asume una disposición subhorizontal (e.g., Jordan et al., 1983). Éste se caracteriza por la ausencia de volcanes activos y por una intensa actividad sísmica (e.g., Cahill e Isacks, 1992) atribuida a la presencia de importantes fallas activas que afectan a los depósitos aluviales cuaternarios, generando cabalgamientos notables de las secuencias neógenas sobre esos depósitos. De esta forma, numerosas evidencias de deformaciones cuaternarias han sido descritas a estas latitudes (Perucca et al., 1999; Costa et al., 2000; Perucca et al., 2012, 2014a, b; 2015; Perucca y Vargas, 2014; Audemard et al., 2016; Rimando et al., 2019, entre otros).

La provincia geológica Precordillera es un orógeno paleozoico que ha sido subdividida, según su estilo estructural en Precordillera Occidental, Central y Oriental (Ortiz y Zambrano, 1981). El área de estudio se ubica en el ámbito de Precordillera Central, que constituye una faja plegada y corrida de carácter epidérmico, con una marcada vergencia hacia el este (Zapata y Allmendinger, 1996; Ramos, 1999), establecida sobre rocas sedimentarias paleozoicas como resultado de la migración hacia el este del frente orogénico (Ramos, 1988), con cordones montañosos que se desarrollan a lo largo de toda la Provincia de San Juan con rumbo N-S. Estos corrimientos forman un abanico imbricado hacia el antepaís (Boyer y Elliot, 1982) donde las láminas imbricadas antiguas se disponen sobre las más nuevas produciendo un aumento progresivo de las inclinaciones originales de las fallas hacia el oeste e incluso el vuelco de los planos de corrimiento (von Gosen, 1992). A pesar del aumento de la inclinación de las fallas, los ángulos de corte de las mismas se mantienen entre $20^{\circ}$ y $30^{\circ} \mathrm{O}$. Esto implicaría que los 
ángulos actuales de las fallas son secundarios y adquiridos por una rotación diferencial que se incrementa hacia el oeste (von Gosen, 1992). A este esquema hay que adicionar la existencia de corrimientos fuera de secuencia como lo es el sistema de falla La Cantera, que constituye un conjunto de corrimientos de bajo ángulo que dispone rocas del Neógeno de la Formación Pachaco sobre depósitos aluviales del Pleistoceno tardío a Holoceno tardío. Este sistema se ubica al oeste de la zona de estudio, sobre el piedemonte occidental de la sierra de La Cantera y su actividad tectónica es al menos holocena (Perucca et al., 2014a, b; 2015).

\section{Metodología}

El análisis geomorfológico y estructural se realizó utilizando datos topográficos, trabajo de campo e imágenes satelitales digitales (Landsat 7-TM y SPOT 5). Se utilizó la hoja geológica publicada por el Servicio Geológico Minero de Argentina (Furque et al., 1998) a escala de 1: 250000 para determinar la litología que se cartografió en la pantalla en un entorno GIS (en formato vectorial) y luego se verificó mediante el trabajo de campo.

La determinación del orden de la cuenca se basó en la metodología propuesta por Strahler (1964). La longitud del cauce principal (Lcp) y la longitud de la cuenca (L), fueron obtenidas de acuerdo con Schumm (1956). La delimitación de la cuenca y el cálculo de los parámetros morfométricos fueron realizados mediante el uso de Google Earth Pro y de SAGA GIS. Los parámetros morfométricos fueron divididos en parámetros básicos tales como: área $(A)$, perímetro $(P)$, longitud $(L)$, orden de la cuenca $(\mathrm{On})$, altura máxima y mínima $(\mathrm{H}, \mathrm{h})$, longitud total de cauces (Ltc), longitud de cauce principal (LCp); y parámetros derivados, obtenidos a partir de las siguientes ecuaciones:

- Relación de elongación: $\operatorname{Re}=(\sqrt{ }(4 \mathrm{~A} / \pi)) / L$ (Schumm, 1956)

- Factor de forma: $\mathrm{Ff}=A / L^{2}$ (Horton, 1932)

- Sinuosidad del cauce: $S=L c p / L$ (Schumm, 1977)

- Ancho medio: $\mathrm{Wm}=A / L$

- Desnivel de cuenca: $\mathrm{Hr}=\mathrm{H}-r$ (Hadley y Schumm, 1961)

- Razón de relieve: $\mathrm{Rr}=\mathrm{Hr} / \mathrm{L}$ (Schumm, 1956)

- Densidad de drenaje: $\mathrm{Dd}=L \mathrm{tc} / \mathrm{A}$ (Horton, 1932)

- Coeficiente de compacidad: $\mathrm{Kc}=0,28 \times \mathrm{p} / \sqrt{ } A$

- Relación de circularidad: $\mathrm{Rci}=4 \pi A / p^{2}$

Por otro lado, los índices morfotectónicos se incluyen entre las técnicas morfométricas y se desarrollaron como una herramienta de reconocimiento básico para identificar áreas que experimentan rápida deformación tectónica. Se calculan a partir de información topográfica, de fotografías aéreas y de modelos digitales de elevación (DEM) (Keller y Pinter, 2002). 
Otro de los índices geomorfológicos utilizados en este trabajo para analizar la evidencia de tectónica activa es la sinuosidad del frente montañoso (Smf) y la asimetría de la cuenca de drenaje (AF). Los índices son combinados, con otra información como tasas de levantamiento a partir de dataciones, para clasificar los grados de actividad tectónica relativa de un área (Keller y Pinter, 2002).

La elaboración del mapa geomorfológico permitió integrar todos estos datos y caracterizar la geomorfología del sector y las estructuras que afectan a ambos piedemontes. Las tareas de campo consistieron en un análisis de las exposiciones naturales identificadas en los cauces que atraviesan las fallas Las Crucecitas y Talacasto occidental, a fines de conocer la cinemática de la falla y los niveles aluviales afectados; lo último para definir, la edad relativa de actividad de los accidentes tectónicos.

Por otra parte, se cubrió el área bajo estudio con la base de datos de gravedad obtenida a partir del WGM12 (World Gravity Map, 2012). El WGM es el primer conjunto de mapas de anomalías de gravedad global que tienen en cuenta un modelo realista de la Tierra y que considera la contribución de la mayoría de las masas superficiales (atmósfera, tierra, océanos, mares interiores, lagos, capas de hielo y plataformas de hielo (Bonvalot et al., 2012). El WGM denota un conjunto de mapas de anomalías de gravedad de alta resolución y cuadrículas digitales computadas a escala global a partir de los modelos disponibles de gravedad de la Tierra y modelos de elevación de referencia. La versión 1.0 (WGM12) incluye un conjunto de tres mapas de anomalías (anomalía de aire libre, de Bouguer completa e isostática) derivado del modelo de gravedad global EGM2008 y el modelo de relieve global ETOPO1 (Bonvalot et al., 2012).

La información de gravedad utilizada se deriva del modelo EGM2008, que fue desarrollado en armónicos esféricos hasta el grado 2160 por la Agencia Nacional de Inteligencia Geoespacial (NGA). El modelo EGM2008 incluye mediciones de gravedad de superficie (a partir de estudios terrestres, marinos o aéreos), altimetría satelital y gravimetría satelital (Misión GRACE). Las anomalías de gravedad se utilizan para investigar la distribución de densidad de masa del interior de la Tierra para proporcionar más claridad en el reconocimiento de las estructuras geológicas en el subsuelo, desde la corteza hasta las profundidades del manto superior (Bonvalot et al., 2012).

La anomalía del aire libre se calculó en la superficie de la Tierra en el contexto de la teoría de Molodensky (Heiskanen y Moritz, 1967) e incluye correcciones para la masa de la atmósfera. La anomalía de Bouguer completa resultante, refleja la perturbación entre la gravedad observada y la calculada para un modelo de Tierra de referencia dado en un punto particular P. También se define a partir de la anomalía de aire libre $(\triangle \mathrm{gFA})$. La anomalía esférica completa de Bouguer se determina en toda la Tierra al calcular en un sólo paso la contribución de la gravedad de todas las masas superficiales por encima o por debajo de la superficie media del mar (Balmino et al., 2011). La anomalía de la gravedad 
esférica de Bouguer en un punto P de la superficie de la Tierra (Balmino et al., 2011) viene dada por:

$$
\Delta \mathrm{gB}=\Delta \mathrm{g}_{\text {surface free-air }}-\Delta \mathrm{g}_{\text {surface masses }}=\mathrm{g}_{\mathrm{obs}}(\mathrm{P})-\gamma(\mathrm{Q})+\delta \mathrm{g}_{\mathrm{FC}}-\delta \mathrm{g}_{\mathrm{TOP}}+\delta \mathrm{g}_{\text {Atm }}
$$

De la misma manera, la contribución de su compensación en el límite cortezamanto también se calcula en geometría esférica sobre la base del equilibrio isostático (modelo Airy-Heiskanen) para determinar la correspondiente anomalía isostática. Se utilizó un enfoque armónico esférico para proporcionar cálculos globales homogéneos y precisos de las correcciones de gravedad y anomalías hasta el grado 10800 (resolución espacial equivalente de media longitud de onda de $\left.1 \times 1^{\prime}\right)$. Los mapas de aire libre, Bouguer y anomalía isostática se cuadraron de acuerdo con el método de curvatura mínima con una grilla de $1.5 \mathrm{~km}$, utilizando el software Oasis Montaj. De acuerdo con la propuesta de Hinze, se consideró una densidad de $2.67 \mathrm{~g} / \mathrm{cm}^{3}$ para las rocas de la corteza superior. Los datos de gravedad cubren la región analizada y sus sectores limítrofes. Para eliminar los efectos de borde, se realizó una expansión de datos. Es bien sabido que la anomalía de Bouguer contiene la suma de los efectos gravimétricos de diferentes fuentes y existen muchas técnicas que permiten una separación adecuada de los mismos. El mapa de anomalías de Bouguer contiene dos efectos: uno regional (atribuido a la corteza inferior y el manto) y otro residual (atribuido a la corteza superior e intrabasamento. Para separar las anomalías de la gravedad regional de las residuales, asumimos que una anomalía regional es causada por fuentes que tienen una amplia extensión horizontal, son profundas en términos de la profundidad hasta la parte superior de las fuentes, y tienen una profundidad similar. Del mismo modo, se asume que una anomalía residual es producida por fuentes que están limitadas en extensión horizontal, son poco profundas y que tienen profundidades diferentes de las anomalías profundas pero similares entre sí.

Para obtener el mapa de anomalías de Bouguer regional, los datos de la grilla se filtraron con un pasabanda (Band pass), rechazando todas las longitudes de onda superiores a $11 \mathrm{~km}$ e inferiores a $3 \mathrm{~km}$. Se obtuvo un gráfico de anomalías residuales que resta la anomalía regional estimada obtenida de la anomalía de Bouguer completa.

\section{Marco geológico}

La unidad más antigua que aflora en el área de estudio (Figura 1c) corresponde a la unidad carbonática integrada por calizas macizas, calizas lajosas y margas de edad tremadociana superior a llanvirniana inferior. Por encima se ubican areniscas y lutitas de edad silúrica. El Devónico está representado por lutitas y areniscas dispuestas en discontinuidad estratigráfica sobre las rocas del Silúrico. Éstas son cubiertas, en discordancia erosiva o en paraconcordancia, por depósitos 
carboníferos, unos $100 \mathrm{~m}$ de conglomerados y areniscas de tonalidades verdes grisáceas (Furque, 1983).

El Neógeno en el área de estudio está expuesto en ambos piedemontes del área de estudio y con un espesor estimado en 1000 m, constituido por depósitos eólicos y de ambiente aluvial y fluvial, con areniscas finas a medianas y limolitas intercaladas con conglomerados. Levina et al. (2014) señalaron edades entre $24.0 \pm 1.2$ Ma y $14.0 \pm 1.6$ Ma para estos depósitos.

En el piedemonte oriental de la sierra de La Crucecita de este a oeste, afloran estratos neógenos con un rumbo $\mathrm{N} 50^{\circ} \mathrm{E}$ y buzamiento $10^{\circ} \mathrm{O}$, que hacia el oeste aumentan a $45^{\circ} \mathrm{O}$ hasta inclinar $70^{\circ} \mathrm{E}$, formando un sinclinal fallado en su flanco occidental por una falla inversa con azimut $160^{\circ}$ y buzamiento $47^{\circ}$ a $67^{\circ} \mathrm{O}$ que levanta las rocas del Devónico sobre rocas del Neógeno (Figura 1c).

En el piedemonte occidental de la sierra de Talacasto, los estratos del Neógeno poseen un rumbo variable de $\mathrm{N} 4^{\circ} \mathrm{E}, \mathrm{N} 16^{\circ} \mathrm{E}$ y $\mathrm{N}-\mathrm{S}$ e inclinan entre 40 y $45^{\circ} \mathrm{O}$, en las proximidades al río de La Burra. Hacia el oeste, el rumbo de los estratos es $\mathrm{N} 8^{\circ} \mathrm{O}$ y el buzamiento aumenta a $70^{\circ} \mathrm{O}$ hasta cubrir en discordancia angular las rocas de edad devónica, con azimut $355^{\circ}$ y buzamiento $60^{\circ} \mathrm{O}$ por la elevación de la sierra de Talacasto que habría generado el basculamiento de toda la cuenca neógena (Figura 1c).

Por encima de los estratos neógenos y en discordancia erosiva se colocan los depósitos aluviales pleistocenos y holocenos, constituidos por fanglomerados y conglomerados mal seleccionados, con clastos angulosos a subangulosos de diferentes tamaños provenientes de las sedimentitas paleozoicas y cenozoicas. Su matriz es arenosa, de granulometría gruesa y frecuentemente la sucesión tiene intercalaciones lenticulares de areniscas gruesas.

Finalmente se mencionan los depósitos actuales de los cauces fluviales provenientes de los diferentes cordones y que se dirigen hacia el río de La Burra, similares a los anteriormente descritos (Figura 1c).

\section{Marco geomorfológico}

En el sector de estudio se identificaron tres unidades morfoestructurales principales, montañosa, de transición y deprimida (Figura 2).

La Unidad Montañosa está conformada por los relieves positivos elevados por falla de las sierras de La Crucecita por el oeste y Talacasto por el este.

La sierra de La Crucecita posee un rumbo NNE con sus mayores elevaciones en el sector central (2 $024 \mathrm{msnm}$ ), y $5 \mathrm{~km}$ de ancho promedio. Constituye un importante relieve positivo que aporta material detrítico hacia el sector este. Este cordón está constituido por rocas sedimentarias del Paleozoico y se caracteriza por tener una pendiente suave en su ladera occidental, mientras que la oriental posee una morfología abrupta debido a la presencia de la falla que levanta las secuencias del Devónico superior por sobre el Neógeno. 


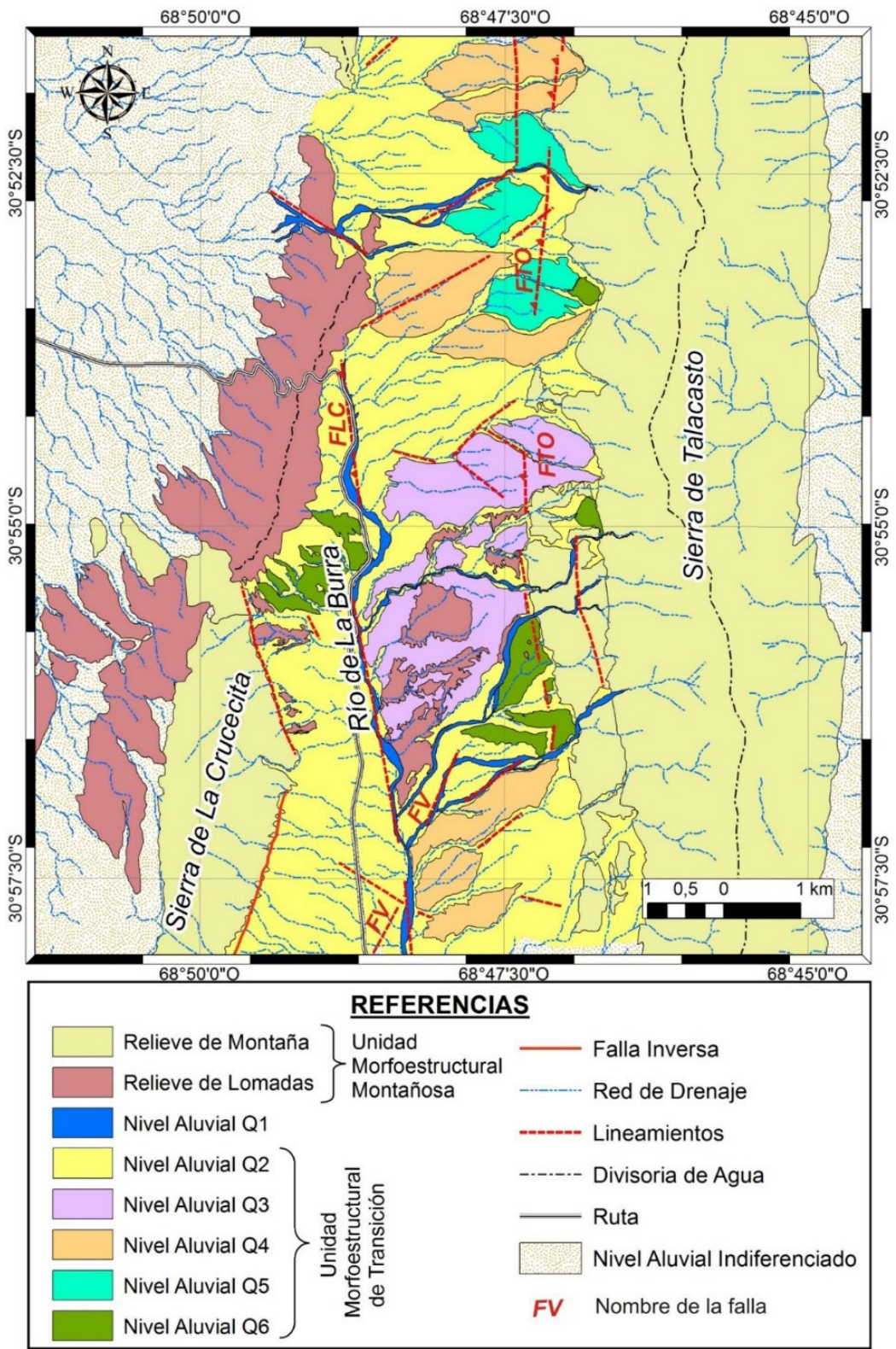

Figura 2. Mapa geomorfológico del área de estudio. Se señalan las tres fallas cuaternarias principales reconocidas: Falla Las Crucecitas, FLC; Falla Talacasto Occidental, FTO; y Falla Vertientes, FV. 
en su porción central la pendiente promedio es de $12.5 \%$, y en el sector sur la pendiente alcanza apenas un 7\% promedio, con una extensión mayor que los sectores anteriores. Por otro lado, el piedemonte occidental de la sierra de Talacasto posee un ancho promedio de $2 \mathrm{~km}$, que queda reducido a menos de $500 \mathrm{~m}$ en su extremo sur, con una pendiente que varía entre $10 \%$ en la parte norte y $5 \%$ en la porción central y sector sur.

El piedemonte está formado por abanicos aluviales que se forman por la acumulación de detritos a partir de los materiales procedentes de la erosión de los altos estructurales (relieve montañoso y de lomadas). En la formación de los mismos el clima representa un papel esencial, ya que en regiones de clima húmedo son poco frecuentes, mientras que en las zonas de clima árido, la escasez de la vegetación sobre el relieve permite la disgregación de éste en fragmentos que se acumulan y extienden al pie de los macizos. Los niveles aluviales sobreyacen a sedimentitas glaciplanizadas del Neógeno, destacándose las superficies tope de las unidades cuaternarias más antiguas por encontrarse también planizadas formando glacis o rampas detríticas.

En ambos piedemontes se identificaron cinco niveles aluviales en función de su posición topográfica, grado de incisión y presencia de barniz del desierto, además y por encima del nivel actual. El nivel más antiguo fue identificado como Q5 y el nivel actual como Q1 (Figura 2). Sin embargo, estos cinco niveles no se desarrollan a lo largo de todo el piedemonte oriental de la sierra de La Crucecita. Por ejemplo, los mismos son claramente identificables en la porción norte donde aflora el Neógeno. Este cambio se produce en coincidencia con una fuerte inflexión del frente montañoso de la sierra controlado por fallas, desde un rumbo $\mathrm{N} 20^{\circ} \mathrm{E}$ a N $20^{\circ} \mathrm{O}$. Hacia el sur de los $30^{\circ} 56^{\prime}$, solamente se reconocen los niveles Q1 y Q2 (Figura 2).

El diseño de la red de drenaje para la cuenca del río Talacasto es subdendrítico y presenta en algunos sectores, evidencias de control estructural (Figura 3a, b). Se asume en general la presencia en el área de materiales homogéneos, no consolidados o suelos. El control estructural de algunos tributarios se manifiesta por ángulos de confluencia rectos, el cual se desarrolla siguiendo estratificación, fallas, fracturas y diaclasas.

Se observa un alto grado de integración ya que, si se eligen dos puntos cualesquiera del sistema, pueden vincularse entre sí sin necesidad de abandonar los cauces.

La cuenca del río Talacasto es de $6^{\circ}$ orden según la clasificación de Strahler (1964; Figura 3a). Los ríos de orden 1, 2, 3 y el río principal (antecedente) de orden 6 presentan orientación E-O, mientras que los cauces de orden 4 y 5 tienen una marcada orientación N-S, paralela a los principales cordones montañosos, elevados por falla. Esta cuenca cubre una superficie (A) de aproximadamente $269.63 \mathrm{~km}^{2}$, valor que permite considerarla como una cuenca grande (Villon, 2002). Además posee un perímetro (P) de 97.09 km, una longitud 

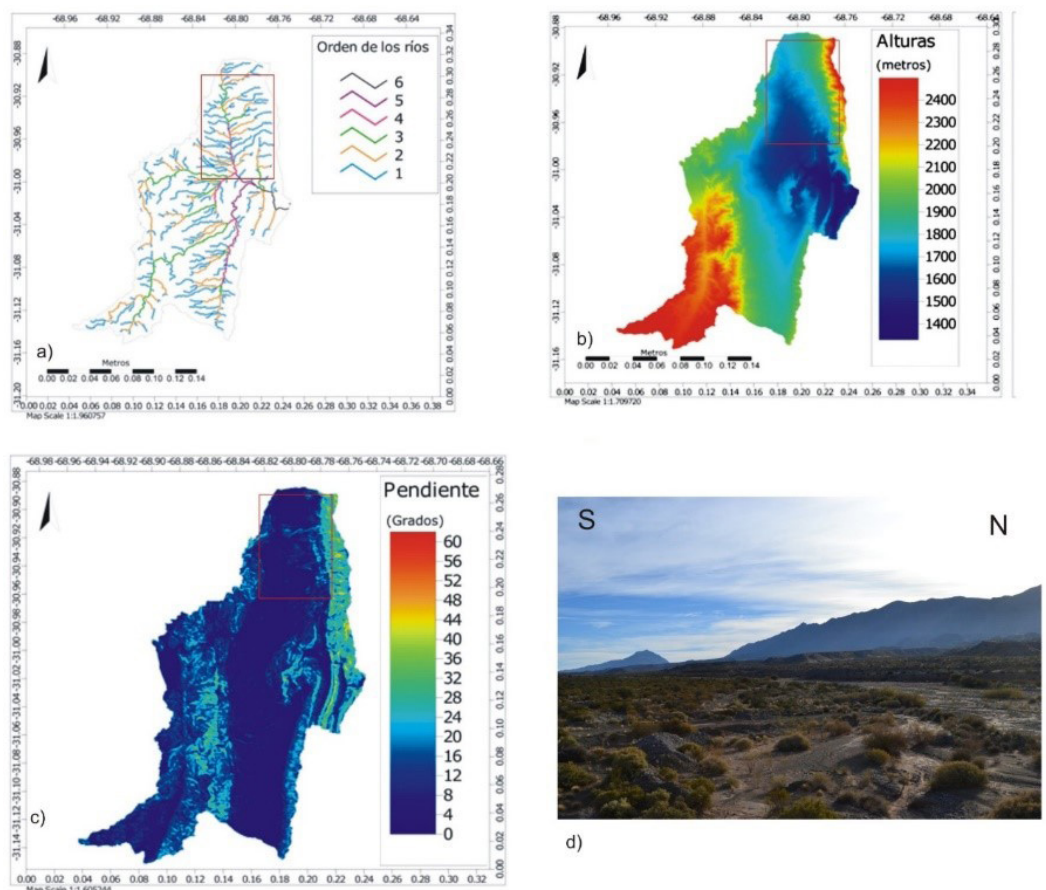

d)

Figura 3. a) Cuenca del río Talacasto y número de orden (el recuadro rojo identifica la ubicación de la Figura 2), b) Mapa de elevaciones, c) Mapa de pendientes, d) Vista al suroeste del valle del río de La Burra.

total de $23.17 \mathrm{~km}$ y un ancho medio $(\mathrm{Wm})$ de $11.63 \mathrm{~km}$. La longitud de su cauce principal (Lcp) es de $37.15 \mathrm{~km}$ mientras que la longitud total de todos sus cauces (Ltc) es de $849.09 \mathrm{~km}$.

Las elevaciones en la cuenca (Figura 3b) muestran valores mínimos entre 1400 m snm y una cota máxima de más de 2400 m snm correspondiente a la sierra de Talacasto al NE y a la sierra de La Crucecita al SO. La cuenca del río Talacasto presenta pendientes máximas (Figura 3c) de hasta $60^{\circ}$ en el flanco oriental de la sierra de Talacasto, y algo menores en el oriental de la sierra de La Crucecita.

Por otra parte, la red de drenaje identificada en ambos piedemontes del río de La Burra es dendrítica divergente, típica de abanicos aluviales. Sin embargo, se producen anomalías en el patrón del drenaje cuando los arroyos atraviesan la traza de las fallas, aumentando el grado de incisión y sinuosidad de los cauces.

La unidad deprimida más importante de la zona, es la planicie aluvial del río de la Burra que es el colector principal de los ríos y arroyos provenientes de los cordones serranos, con un rumbo N-S (Figuras 1 y 2). En la margen izquierda de 
este río se ubican formas aluviales relícticas tales como tres terrazas de erosión y acumulación ubicadas a diferentes alturas, siendo las más antiguas las que están topográficamente más altas y se encuentran distribuidas asimétricamente (Figura 2). Su origen puede ser debido a cambios climáticos o a la variación de nivel de base por causas tectónicas. Si bien es posible que haya una combinación de ambos procesos, en la porción norte de la depresión se infiere una causa tectónica, debido a la evidencia de actividad tectónica cuaternaria identificada en ambos piedemontes (Figura 2).

El factor de asimetría fue desarrollado para detectar basculamientos tectónicos sobre cuencas de drenaje en áreas extensas (Keller y Pinter, 2002). El valor obtenido para la cuenca de Talacasto es $36.31 \%$, correspondiendo a una cuenca marcadamente asimétrica, lo que sugiere un basculamiento de la cuenca hacia el este, en coincidencia con el avance del frente orogénico (Figura 3a, b, c).

La sinuosidad del frente de montaña refleja el balance entre las fueras erosivas de los ríos que tienden a formar engolfamientos al salir del sector montañoso y las fuerzas tectónicas que tienden a producir un frente recto coincidente con una falla activa. La cuenca en estudio, presenta un índice de sinuosidad del frente montañoso de 0,97 , indicando frentes controlados por estructuras activas. A mayor actividad tectónica, la unión piedemonte-montaña tenderá a coincidir con la traza de la falla, dando un índice cercano a 1.

\section{Análisis neotectónico}

Se identificaron fallas con evidencia de actividad tectónica cuaternaria en ambos piedemontes del valle del río de La Burra. Todas las fallas analizadas poseen rumbo submeridianal, con componente de movimiento principal vertical, inversas y con vergencia oriental, consistente con el estilo estructural de Precordillera Central (Figura 2).

Estas estructuras fueron denominadas de norte a sur: Las Crucecitas y Vertientes (piedemonte oriental de La Crucecita) y Sistema de falla Talacasto occidental, ubicado en el piedemonte occidental de la sierra homónima. Las fallas afectan los depósitos asignados al Pleistoceno tardío y Holoceno y se definen por una serie de geoformas típicas de ambientes compresivos tales como escarpas a favor (piedemonte occidental) y en contra de la pendiente (piedemonte oriental), terrazas restringidas al bloque elevado de las fallas, lagunas de fallas (sagponds), vertientes alineadas, drenajes obturados, desviados, fuertemente incisos y sinuosos en los bloques elevados de las fallas, abanicos aluviales con pendientes anómalas, entre otros rasgos que señalan su actividad tectónica cuaternaria. Se identificaron en función de la posición relativa e incisión, así como por la presencia de pavimento y barniz del desierto, cinco (5) niveles aluviales cuaternarios en el sector norte del piedemonte oriental de la sierra de La Crucecita, denominados del más reciente al más antiguo: Q1, Q2, Q3, Q4 y $\mathrm{Q} 5$; y probablemente hasta un sexto nivel (Q6) en el piedemonte occidental de 
la sierra de Talacasto. En este análisis se determinó que las fallas afectan todos los niveles aluviales (a excepción del actual Q1) y coinciden con el estilo estructural de Precordillera Central, inversas de bajo ángulo y con vergencia oriental. Esta deformación se vincula con el tectonismo que a escala regional afectó y afecta al ámbito precordillerano.

\section{Piedemonte Oriental sierra de La Crucecita}

De norte a sur, existen notorias diferencias geomorfológicas a lo largo de este piedemonte. En su porción norte, se han reconocido cuatro niveles aluviales fuertemente incisos que cubren las rocas del Neógeno y han sufrido procesos de glaciplanación (Figura 2).

Hacia el sur, solamente se han identificado el nivel actual $\left(Q_{1}\right)$ y el nivel Q2; y solamente en los sectores afectados por la falla Las Vertientes, afloran rocas del Neógeno.

Sobre el extremo norte del piedemonte oriental de la Sierra de la Crucecita se ubica una falla inversa, denominada falla Las Crucecitas, que afecta el nivel aluvial Q4 con escarpa al este suavemente redondeada, de aproximadamente $1.70 \mathrm{~m}$ de altura, así como una terraza de erosión de un tributario del río de La Burra en la que la falla posee azimut $30^{\circ}$ y un buzamiento de $40^{\circ} \mathrm{O}$. Coloca sedimentitas neógenas de la Formación Cuculí sobre depósitos aluviales asignados en este trabajo tentativamente al Holoceno (Figuras $4 a$ y b).

Hacia el sur, otra exposición natural muestra la falla Las Crucecitas con azimut $20^{\circ}$ e inclinación $40^{\circ} \mathrm{O}$ (Perucca y Vargas, 2014). Se midieron estrías en el plano de falla, cuyo pitch es de $57^{\circ} \mathrm{NO}$, indicando un movimiento vertical con componente horizontal sinestral. La falla coloca rocas del Neógeno sobre depósitos cuaternarios del nivel Q4 asignados tentativamente al Pleistoceno; y aunque no corta la superficie, presenta la superficie combada, correspondiendo a un cabalgamiento ciego (Figura 4a). Esta suave inflexión de la superficie (escarpe de flexura) del nivel aluvial constituye un marcador planar deformado y flexurado que evidencia la presencia de la falla, aunque la misma no aflore en superficie. Los fanglomerados del nivel Q4 presentan lentes de materiales finos como así también clastos orientados y flexurados como resultado de la deformación.

En la porción centro y sur del piedemonte oriental de la sierra de La Crucecita, se destaca una falla con rumbo promedio $24^{\circ} \mathrm{con}$ una suave inflexión en la parte sur donde cambia a $11^{\circ}$ (Figura 4c, d), con el labio hundido al este, cuyo plano de falla inclinaría al oeste (consistente con la estructura regional de Precordillera Central). Esta falla, denominada falla Vertientes, desplaza depósitos aluviales asignados al Pleistoceno tardío-Holoceno del nivel Q2. La longitud de la falla es de $\sim 6 \mathrm{~km}$ y afecta también la porción distal del piedemonte occidental de la sierra de Talacasto, donde eleva rocas del Neógeno con rumbo $\mathrm{N} 10^{\circ}$ e inclinación $45^{\circ}$ al $\mathrm{O}$ (Figura 2). Si bien no se han encontrado exposiciones naturales que indiquen su cinemática se infiere su carácter inverso y vergencia al este. 

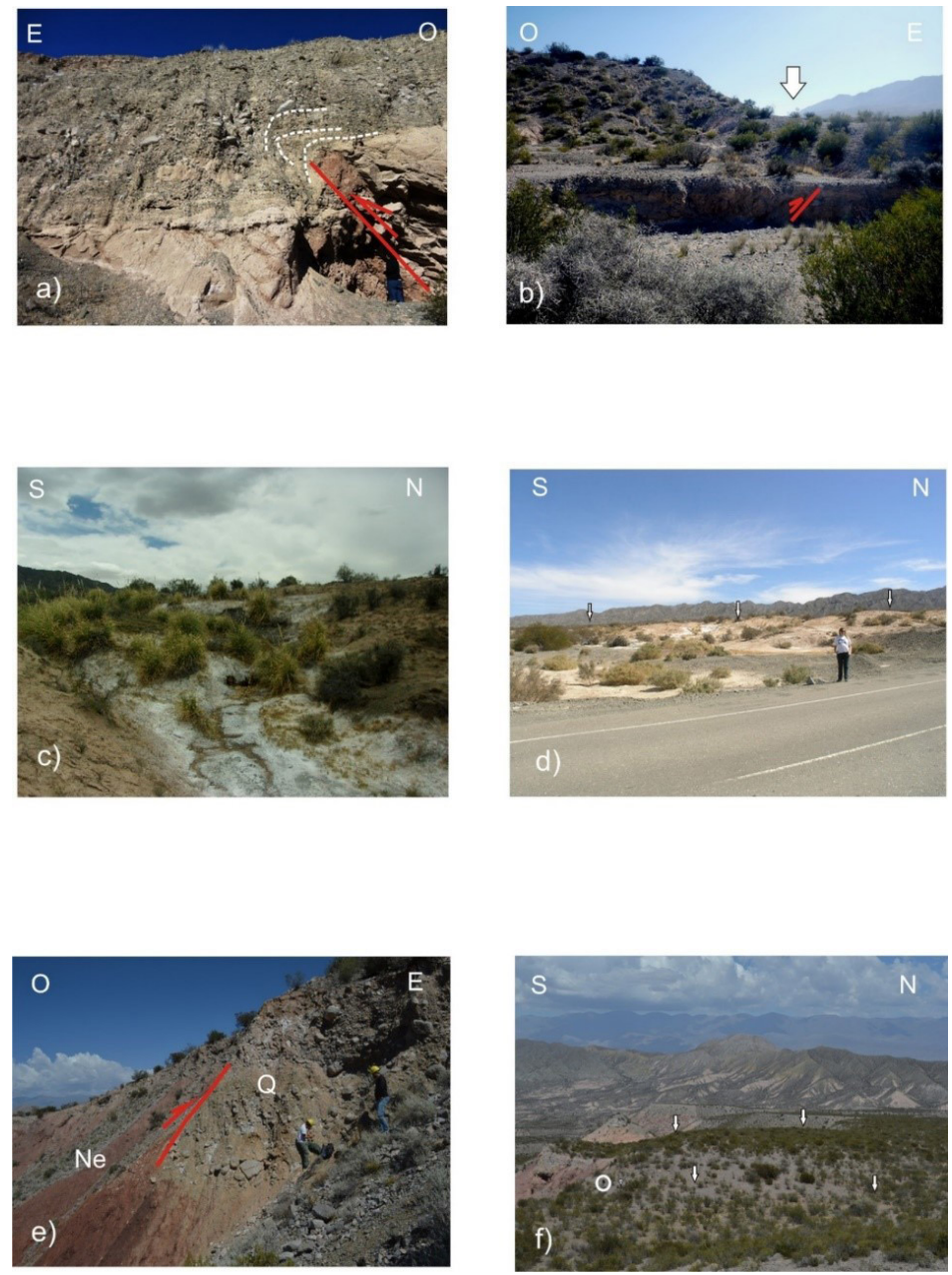

Figura 4. a) Vista hacia el sur de exposición natural en la que se aprecia la falla inversa Las Crucecitas, que inclina al oeste y coloca rocas del Neógeno sobre depósitos aluviales pleistocenos del nivel Q3. La falla no corta la superficie pero deforma los niveles aluviales más antiguos, b) Exposición natural donde se aprecian depósitos probablemente holocenos de una terraza ubicada sobre la margen izquierda de un pequeño tributario del río de La Burra. Al fondo, se puede apreciar una escarpa de flexura de la falla Las (rucecitas preservada en el nivel aluvial Q3, redondeada y de aproximadamente $3 \mathrm{~m}$ de altura, c) Vista al oeste de los depósitos limosos y vertientes alineadas a lo largo de la falla, d) Depósitos finos asociados a las vertientes, con salida de agua permanente, e) Vista hacia el norte de la falla Talacasto Occidental. La falla coloca rocas del Neógeno sobre depósitos aluviales cuaternarios. f) Vista al oeste de la escarpa a contrapendiente de la falla Talacasto Occidental, señalada por flechas. El círculo blanco señala una persona a modo de escala. 


\section{Piedemonte Occidental sierra de Talacasto}

En el piedemonte occidental de la sierra de Talacasto se identificaron secciones de falla discontinuas y subparalelas que fueron identificadas a partir de escarpes de fallas a contrapendiente, simples y compuestos, terrazas en el bloque elevado, escarpes flexurales, plegamiento reciente en depósitos cuaternarios y diversas anomalías en la red de drenaje. Además se observa un aumento en la incisión y sinuosidad de los cauces en el bloque elevado de la falla, donde se exponen las rocas del Neógeno. La altura de los escarpes de falla varía entre $0.30 \mathrm{~m}$ y $10 \mathrm{~m}$, encontrándose las mayores elevaciones en la porción norte del piedemonte. La falla coloca rocas del Neógeno sobre depósitos fluviales del nivel Q4, inclina al oeste $74^{\circ}$ y su azimut es $25^{\circ}$ (Figura $4 \mathrm{e}, \mathrm{f}$ ).

\section{Análisis gravimétrico}

Las cartas de anomalías obtenidas para el área de La Burra reflejan contrastes de alta densidad a escala de la corteza y la litosfera, donde los valores positivos y negativos se asocian aparentemente a las diferentes unidades expuestas en el área de estudio. Es importante resaltar la fuerte influencia de la raíz andina sobre las anomalías observadas. El mapa de anomalías de aire libre (Figura 5a) muestra la correspondencia con los frentes inversos que elevan las distintas sierras de la Precordillera Central, es decir, la sierra de Talacasto al este y la sierra de la Crucecita al oeste. Además, los cambios de gradiente observados entre las unidades morfotectónicas elevadas al este (Sierra de Talacasto) y al oeste (Sierra de la Crucecita) limitarían la depresión correspondiente al valle de La Burra.

En la carta de anomalías de Bouguer completa, se observaron valores negativos asociados con las cadenas montañosas que exhiben unidades paleozoicas y que fueron elevadas por fallas N-S (Figura 5b). Por otro lado, los valores positivos parecen mostrar la existencia de depocentros entre las montañas (valle de La Burra) donde predominan los depósitos Neógenocuaternarios. Estos valles parecen estar controlados por estructuras NE y NO.

La carta de anomalías isostáticas sugiere preliminarmente un fuerte desequilibrio para la región en estudio, evidenciada por valores negativos a lo largo de las principales cadenas montañosas tal como en la sierra de Talacasto, al este del sector analizado. Por otro lado, los depocentros cenozoicos muestran valores positivos que también sugieren un desequilibrio isostático, por lo que se esperarán ajustes tectónicos para la región analizada para poder alcanzar el equilibrio isostático (Figura 5c).

Por su parte, el análisis de las cartas de anomalías residuales sugiere la existencia de controles estructurales (frentes de empuje) con rumbo $\mathrm{N}-\mathrm{S}$ que exponen las unidades del Paleozoico, alcanzando las mayores elevaciones topográficas. Estas estructuras limitan tanto el este como el oeste por valles intermontanos rellenos por sedimentos del Neógeno a Cuaternario. Además, la carta de anomalías residuales revela contrastes laterales de densidad en 

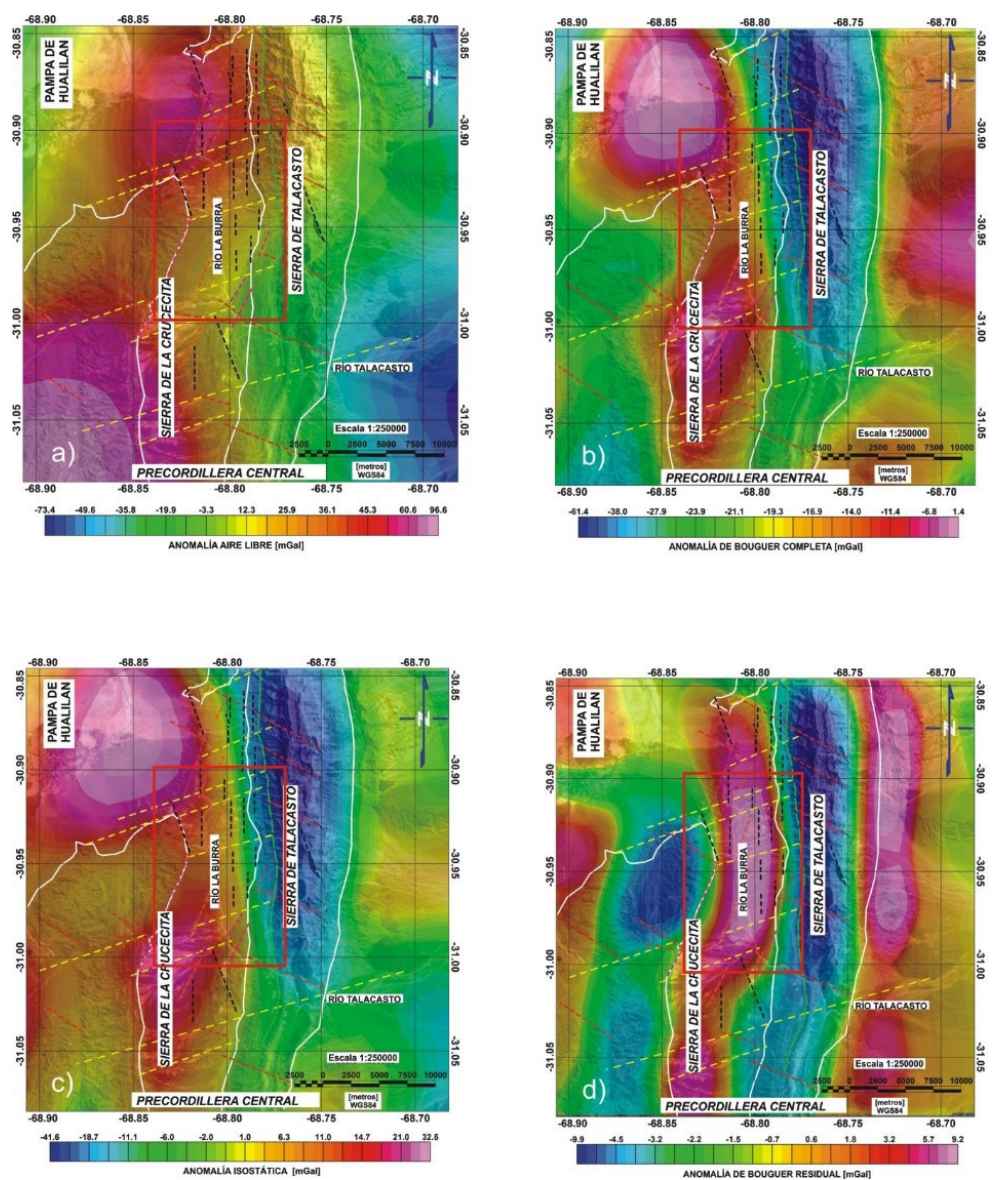

Figura 5. a) Mapa de anomalías de aire libre, b) mapa de anomalías de Bouguer completas, c) mapa de anomalías isostáticas, d) mapa de anomalías de Bouguer residuales. Los recuadros rojos indican el área de estudio en el valle del río de La Burra.

dirección norte-sur asociados a las estructuras NE y NO que segmentarían los valles intermontanos (Figura $5 \mathrm{~d}$ ).

\section{Discusión y conclusiones}

Numerosas evidencias de actividad tectónica cuaternaria se desarrollan en el valle longitudinal ubicado entre la sierra de La Crucecita, por el oeste y la sierra de Talacasto por el este, en el ámbito del cinturón plegado y cabalgado de la Precordillera Central. 
La identificación de la existencia de deformaciones activas a lo largo de ambos piedemontes, las cuales son observables tanto en fotografías aéreas como en el campo, muestran una orientación preferencial N-S, a excepción de la falla

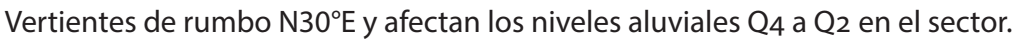

Intepretamos que los niveles Q6-Q3 pudiesen ser tentativamente asignados al Pleistoceno tardío, mientras que los niveles Q2 hasta el nivel actual serían del Holoceno. Del análisis de las geoformas en ambos piedemontes, es posible determinar el control estructural que ejercen estas estructuras y su actividad tectónica holocena. Todas ellas mostraron cabalgamientos de rumbo casi norte sur y buzamientos entre $40^{\circ}$ y $70^{\circ} \mathrm{O}$. Se reconocieron además pliegues de arrastre y fallas menores de poco desplazamiento. Se considera estas estructuras como fuentes sismogénicas potenciales en la región precordillerana, cercana a la ciudad de San Juan y a presas cimentadas y a construir sobre el río San Juan.

\section{Agradecimientos}

La presente contribución fue financiada con fondos del IPGH, para el proyecto GEOF-03 2018, como así también CICITCA 2016-17 (UNSJ). Los autores agradecen a CIGEOBIO (CONICET-UNSJ) por la entrega de fondos para la obtención de la licencia del software ArcGIS 10.3.

\section{Bibliografía}

Allis, C., 2016. "Geomorfología y Neotectónica en el Piedemonte Oriental de la Sierra de La Crucecita, Precordillera Central, Provincia de San Juan", trabajo final de licenciatura de la Universidad Nacional de San Juan, inédito, 80 pp.

Audemard, F.A., 1999. "Morpho-structural expression of active thrust fault systems in the Humid Tropical Foothills of Colombia and Venezuela", Zeitschrift fur Geomorphologie, 118: 1-18.

Audemard, F.A.; Perucca, L.P.; Pantano, A.; Avila, C.; Onorato, M.R.; Vargas. H.N.; Alvarado, P. \& Viete, H., 2016. "Holocene compression in the Acequión valley (Andes Precordillera, San Juan Province, Argentina): Geomorphic, tectonic, and paleoseismic evidence", J South American Earth Sciences, 67: 140-157.

Balmino, G.; Vales, N.; Bonvalot, S. \& Briais, A., 2012. "Spherical harmonic modeling to ultrahigh degree of Bouguer and isostatic anomalies", Journal of Geodesy, 86: 7: 499520. DOI: https://www.doi.org/10.1007/s00190-011-0533-4

Bonvalot, S.; Balmino, G.; Briais, A.; Kuhn, M.; Peyrefitte, A.; Vales N.; R. Biancale; G. Gabalda; G. Moreaux; F. Reinquin; M. Sarrahilh, 2012. World Gravity Map. Bureau Gravimetrique International (BGI), map, CGMW-BGI-CNES-IRD Ed., Paris.

Boyer, S.E. \& Elliott, D., 1982. "Thrust systems", Bulletin of the American Association of Petroleum Geologists, 66: 1196-1230.

Cahill, T. \& Isacks, B.L., 1992. "Seismicity and the shape of the subducted Nazca plate", Journal of Geophysical Research, 97: 17503-17529. 
Casa, A.; Yamín, M.; Wright, E.; Costa, C.; Coppolecchia, M. \& Cegarra, M., 2011. Deformaciones Cuaternarias de la República Argentina, Sistema de Información Geográfica, Instituto de Geología y Recursos Minerales, Servicio Geológico Minero Argentino, publ. 171, v.1.0 DVD.

Casa, A.; Yamin, M.; Wright, E.; Costa, C.; Coppolecchia, M.; Cegarra, M. \& Hongn, F. (eds.), 2014. Deformaciones cuaternarias de la República. Argentina. Sistema de Información Geográfica, Instituto de Geología y Recursos Minerales, Servicio Geológico Minero. Argentino, publ. 171, v.1.0 en formato DVD.

Costa, C.; Machette, M.N.; Dart, R.L.; Bastías, H.E.; Paredes, J.D.; Perucca, L.P.; Tello, G.E. \& Haller, K.M., 2000. Map and database of Quaternary faults and folds in Argentina, U.S. Geological Survey Open-File Report 00-0108, 81 pp.

Furque, G., 1983. “Descripción Geológica de la Hoja 19 C, Ciénaga de Hualilán”, Boletín de la Secretaría de Minería, 193, Buenos Aires, 111 pp.

Furque, G.; González, P.; Caballé, M., 1998. “Descripción de la Hoja Geológica 3169-II, San José de Jáchal (Provincias de San Juan y La Rioja)", Servicio Geológico y Minero Argentino, Boletín 259, 150 pp.

Hadley, R.F. \& Schumm, S.A., 1961. "Sediment sources and drainage basin characteristics in upper Cheyenne River Basin", U.S. Geological Survey Water-Supply Paper, 1531-B, $198 \mathrm{pp}$.

Heiskanen, W.A. \& Moritz, H., 1967. Physical Geodesy, WH Freeman \& Co, San Francisco, USA, $364 \mathrm{pp}$.

Horton, R.E., 1932. Drainage basin characteristics: American Geophysics Union Trans., 13: 350361.

Jordan, T.E.; Allmendinger, R.W.; Brewer, J.A.; Ramos, V.A. \& Ando, C.J., 1983. "Andean tectonics related to geometry of subducted Nazca plate", Geological Society of America Bulletin, 94: 341-361.

Keller, E.A. \& Pinter, N., 2002. Active Tectonics, Earthquakes, Uplift and Landscape, 2nd Edition, Prentice Hall, Upper Saddle River, 362 pp.

Levina, M.; Horton, B.; Fuentes, F. \& Stockli, D., 2014. "Cenozoic sedimentation and exhumation of the foreland basin system preserved in the Precordillera thrust belt (31-32 ${ }^{\circ}$ S), southern central Andes, Argentina", Tectonics, 33: 1659-1680. DOI: https://www.doi.org/10.1002/2013TC003424.

Ocaña, R., Flores, D., Alcayaga, G., Suvires, G., 2017. "Geomorphology of Talacasto Aluvial Fan, Precordillera of San Juan, Argentina", Journal of Maps, 13:2, 929-935. DOI: https://www.doi.org/10.1080/17445647.2017.1409138.

Ortiz, A. \& Zambrano, J., 1981. "La provincia geológica de Precordillera Oriental”, Proceedings, $8^{\circ}$ Congreso Geológico Argentino, San Luis, 3: 59-74.

Perucca, L.P. \& Vargas, H.N., 2014. “Neotectónica de la provincia de San Juan, centro-oeste de Argentina", Bol. de la Soc. Geol. Mexicana, 66: 291-304.

Perucca, L.; Paredes, J.; Tello, G. \& Bastías, H., 1999. "Fallamiento activo en el área norte del sistema de fallamiento El Tigre, San Juan-La Rioja", Revista de la Asociación Geológica Argentina, 54: 206-214. 
Perucca, L.P.; Lara, G. \& Vargas, N., 2012. "Nueva evidencia de actividad tectónica cuaternaria en la depresión Zonda-Maradona, provincia de San Juan", Revista Asociación Geológica Argentina, 69: 97-105.

Perucca, L.P.; Rothis, M. \& Vargas, H.N., 2014a. "Morphotectonic and neotectonic control on river pattern in the Sierra de la Cantera piedmont, Central Precordillera, province of San Juan, Argentina", Geomorphology, 204: 673-682.

Perucca, L.P.; Onorato, M.; Millán, J.; Bustos, A. \& Vargas, H.N., 2014b. “Variación del estilo de deformación a lo largo del Sistema de falla La Cantera, Precordillera Central, San Juan, Argentina", Revista de la Sociedad Geológica de España, 27(2): 69-79.

Perucca, L.; Rothis, M.; Bezerra, F.; Vargas, N. \& Lima, J., 2015. “Late Quaternary evolution of the La Cantera Fault System (Central Precordillera, Argentina): a morphotectonic and paleoseismic analysis", Tectonophysics.

DOI: https://www.doi.org/10.1016/j.tecto.2015.08.041

Ramos, V., 1988. "The Tectonics of the Central Andes; $30^{\circ}$ to $33^{\circ} \mathrm{S}$ latitude”, Geological Society of America Special Paper, 218: 31-54.

__ 1999. "Las provincias geológicas del territorio Argentino", en Caminos, R. (ed.), Geología Argentina, Instituto de Geología y Recursos Minerales, Anales, 29(12): 41-96.

Rimando, J.M.; Schoenbohm, L.M.; Costa, C.H.; Owen, L.A.; Cesta, J.M.; Richard, A.D., \& Gardini, C.E., 2019. "Late Quaternary Activity of the La Rinconada Fault Zone, San Juan, Argentina", Tectonics. DOI: https://www.doi.org/10.1029/2018TC005321.

Schumm, S.A., 1956. "Evolution of drainage systems and slopes in badlands at Perth Ambos, New Jersey", Geological Society of America Bulletin, 67: 597-646.

Schumm, S.A., 1977. The fluvial system, New York, Jonh Wiley \& Sons, 338 pp.

Soil Conservation Service, 1986. Urban hydrology for small watersheds, TR-55, 2nd ed., USA.

Strahler, A.N., 1964. "Quantitative geomorphology of drainage basin and channel networks", en Chow, V.T. (ed.), Handbook of applied hydrology, New York, McGraw Hill, pp. 4-76.

Villon, 2002. Hidrología, Instituto Tecnológico de Costa Rica, Facultad de Ingeniería Agrícola, 2a. edición, Ediciones Villon, Lima, Perú, 15-64 pp.

Von Gosen, W., 1992. "Structural evolution of the Argentine Precordillera: the Rio San Juan section", J. Struct. Geol., 14(6): 643-667.

Zapata, T.R. \& Allmendinger, R.W., 1996. “Thrust-front zone of the Precordillera, Argentina: a thick-skinned Triangle zone”, Am. Assoc. Petroleum Geol. Bull., 80: 359-381. 